\title{
SOME EXAMPLES OF EXTREMAL TRIPLES OF COMMUTING CONTRACTIONS
}

\author{
EDWARD J. TIMKO
}

Abstract. The collection $\mathfrak{C}_{3}$ of all triples of commuting contractions forms a family in the sense of Agler, and so has an "optimal" model $\partial \mathfrak{C}_{3}$ generated by its extremal elements. A given $T \in \mathfrak{C}_{3}$ is extremal if every $X \in \mathfrak{C}_{3}$ extending $T$ is an extension by direct sum. We show that many of the known examples of triples in $\mathfrak{C}_{3}$ that fail to have coisometric extensions are in fact extremal.

Mathematics subject classification (2010): 47A13, 47A45.

Keywords and phrases: Hilbert space operators; commuting contractions; extremal elements.

\section{REFERENCES}

[1] J. AGLER, An abstract approach to model theory, Surveys of some recent results in operator theory, Vol. II, 1-23, Pitman Res. Notes Math. Ser., 192, Longman Sci. Tech., Harlow 1988.

[2] T. Andô, On a pair of commutative contractions, Acta Sci. Math. (Szeged) 24 (1963) 88-90.

[3] R. CURTO, W. LEE, Towards a model theory for 2-hyponormal operators, Integral Equations Operator Theory 44 (2002), no. 3, 290-315.

[4] M. Dritschel, S. McCullough, Model theory for hyponormal contractions, Integral Equations Operator Theory 36 (2000), no. 2, 182-192.

[5] M. DRitschel, S. MCCUllough, Boundary representations for families of representations of operator algebras and spaces, J. Operator Theory $\mathbf{5 3}$ (2005), no. 1, 159-167.

[6] M. Dritschel, S. McCullough, H. Woerdeman, Model theory for $\rho$-contractions, $\rho \leqslant 2$, J. Operator Theory 41 (1999), no. 2, 321-350.

[7] B. A. LotTo, T. STEGER, von Neumann's inequality for commuting, diagonalizable contractions, II, Proc. Amer. Math. Soc. 120 (1994), no. 3, 897-901.

[8] S. PARrotT, Unitary dilations of commuting contractions, Pacific J. Math. 34 (1970) 481-490.

[9] G. PISIER, Similarity problems and completely bounded maps, Lecture Notes in Mathematics, 1618, Springer-Verlag, Berlin, 1996.

[10] S. Richter, C. SundBerg, Joint extensions in families of contractive commuting operator tuples, J. Funct. Anal. 258 (2010), no. 10, 3319-3346.

[11] B. Sz. NAGY, C. FoIAs, H. BerCovici, L. KéRCHY, Harmonic analysis of operators on Hilbert space, 2nd ed. Revised and enlarged ed. Universitext. Spring, New York 2010. 